Meta

Journal des traducteurs

Translators' Journal

\title{
Histoire de la psychiatrie en traduction : contextes et mouvements de traduction
}

\section{Mariane Gingras Harvey}

Volume 60, numéro 2, août 2015

$60^{\mathrm{e}}$ anniversaire. Les horizons de la traduction : retour vers le futur $60^{\text {th }}$ Anniversary. Translation's Horizons: Back to the Future

60mo aniversario. Los horizontes de la traducción: regreso al futuro

URI : https://id.erudit.org/iderudit/1032888ar

DOI : https://doi.org/10.7202/1032888ar

Aller au sommaire du numéro

Éditeur(s)

Les Presses de l’Université de Montréal

ISSN

0026-0452 (imprimé)

1492-1421 (numérique)

Découvrir la revue

Citer cette note

Gingras Harvey, M. (2015). Histoire de la psychiatrie en traduction : contextes et mouvements de traduction. Meta, 60(2), 337-337.

https://doi.org/10.7202/1032888ar d'utilisation que vous pouvez consulter en ligne. 


\title{
Histoire de la psychiatrie en traduction: contextes et mouvements de traduction
}

\author{
Mariane Gingras Harvey \\ Université de Montréal, Montréal, Canada \\ m.gingras.harvey@umontreal.ca
}

\begin{abstract}
Notre présentation vise à explorer le rôle de la traduction dans la diffusion des savoirs ayant mené à l'établissement d'une nouvelle discipline: la psychiatrie. Celle-ci apparaît en Europe durant la seconde moitié du XVIII ${ }^{\mathrm{e}}$ siècle lorsqu'une corrélation commence à s'établir entre la maladie mentale et certains phénomènes biologiques. Plusieurs savants européens proposent alors des idées novatrices aujourd'hui considérées comme fondatrices du domaine. Associé à la notion de traitement moral, le Français Philippe Pinel est généralement reconnu comme le fondateur de la psychiatrie moderne; son disciple, Jean-Étienne Dominique Esquirol (1772-1840), est principalement reconnu pour sa thèse sur les passions dirigée par Pinel. On doit à Johann Christian Reil (1759-1813) le terme allemand Psychiatrie (vers 1808). Enfin, Thomas Arnold (1742-1816) est l'auteur du premier texte de psychiatrie en Angleterre et Alexander Crichton (1763-1856), son compatriote, s'est penché sur la psychopathologie des passions et ses relations avec les maladies mentales. Ces auteurs, qui ont écrit en français (Pinel et Esquirol), en allemand (Reil) et en anglais (Arnold et Crichton), ont tous été traduits vers au moins l'une ou l'autre de ces trois langues. Notre hypothèse de travail est que les mouvements de traduction se sont révélés essentiels à la fondation de la psychiatrie. Dans le cadre de cette présentation, nous présentons la première étape de notre projet, qui vise à caractériser les principaux écrits de ces auteurs ainsi que leurs traductions: où et quand les textes et les traductions ont été publiés, à partir de quelles langues et vers quelles langues ils sont traduits, qui en sont les auteurs et les traducteurs et quelle a été la réception des originaux et des traductions.
\end{abstract}

Mariane Gingras Harvey poursuit actuellement des études doctorales à l'Université de Montréal, d'où elle détient un baccalauréat et une maîtrise en traduction. Sa thèse, dirigée par madame Sylvie Vandaele, traitera du rôle de la traduction dans l'établissement de la psychiatrie comme discipline entre la fin $d u X v I^{e}$ et le début du XIXe siècle. À ce jour, madame Gingras Harvey a publié deux articles, le premier seule et le second en collaboration avec sa directrice de recherche. Dans les dernières années, elle a présenté deux conférences aux congrès de l'Association canadienne de traductologie (en 2013 et en 2014) ainsi qu'une conférence au congrès de la CERLIS à Bergame en Italie (en 2014). Elle a par ailleurs travaillé comme assistante éditoriale à la revue Meta (entre 2013 et 2014). 